\title{
Numerical Simulation on End Suction Centrifugal Pump Running in Inverse Flow for Microhydro Applications
}

\author{
Mohd Azlan Ismail ${ }^{1, a^{*}}$, Al Khalid Othman ${ }^{2, b}$, Hushairi Zen ${ }^{3, c}$ \\ ${ }^{1}$ Faculty of Engineering, University Malaysia Sarawak, Kota Samarahan, Sarawak, Malaysia \\ ${ }^{2}$ Faculty of Engineering, University Malaysia Sarawak, Kota Samarahan, Sarawak, Malaysia \\ ${ }^{3}$ Faculty of Engineering, University Malaysia Sarawak, Kota Samarahan, Sarawak, Malaysia \\ alanzmr@gmail.com, bokhalid@feng.unimas.my, 'chushair@feng.unimas.my
}

Keywords: computational fluid dynamics; microhydro; pump as turbine; rural electrification; performance curve

\begin{abstract}
The initial capital cost for most microhydro projects has always been an overriding issue for self-funded remote communities. The cost will escalate significantly in the absence of local microhydro electromechanical manufacturers. The application of end suction centrifugal pump as turbine will reduce the overall cost, which renders microhydro systems feasible for self-funded projects and are therefore suitable for rural communities. The goal of this study is to design and develop a pump as turbine (PAT) which serves as a substitute to commercial electromechanical components. Numerical analysis of an inverse flow for an end suction centrifugal pump is presented in this paper, which includes the performance curves and hydraulic characteristics of the pump. ANSYS CFX, a commercial CFD software is used to simulate the performance of the pump with specific speed, $\mathrm{N}_{\mathrm{s}}$ of 70 units (Euroflo EU50-20). The computational flow domain inside the pump is comprises of impeller, volute and draft tube. Unstructured tetrahedral mesh is used to maintain good surface mesh due to complex flow domain geometries. The governing equations used in the simulations are three-dimensional, incompressible Navier-Stokes and $k-\epsilon$ turbulence model under steady-state condition. The simulation results are compared with pump performance curve supplied by the pump manufacturer. The verification results show good agreement for flow rates between 0.7 and 1.3 Q QEeP. The best efficient point (BEP) for inverse flow is attained at a higher head and flow rate compared to pump mode, whereby the value is found to be $21.55 \mathrm{~m}$ and $14.0 \mathrm{l} / \mathrm{s}$, respectively. It is believed that the findings of this study will be useful to predict hydraulic characteristics and performance curves of PAT and the model may be used to identify poor flow characteristics inside the pump. It is recommended that optimization process is carried out using CFD tools in future studies.
\end{abstract}

\section{Introduction}

The use of pump as turbine (PAT) in replacement of commercial electromechanical components has been proven to be a feasible, low-cost solution. A number of successful microhydro projects have been reported for rural area sites, especially in developing countries [1-3]. Even though PAT has lower efficiency than commercial turbines, its availability covers a wide range of flows and heads, which makes microhydro projects more economical and practical. In addition, PAT offers benefits such that it has a simple construction, its spare parts are easily attainable, maintenance services are readily available and installation can be carried out by local laymen. The favourable characteristics of PAT are indeed important as they reduce the dependence on third-party professional services which can be very expensive.

It is known that the performance curve of the pump is different when it is run in turbine mode since its hydraulic characteristics do not match due to the inverse flow through the volute, impeller and draft tube. Pump manufacturers will usually supply the pump performance curve and therefore the pump needs to be tested over a range of flows and heads by means of a hydraulic rig in order to acquire the turbine performance curve. Derakhshan and Nourbakhsh (2008), Raman et al. (2013), 
Fernández et. al (2004), and Nautiyal et al. (2011) presented experimental analysis of PAT for specific speed ranging between 14 to 60 [4-7]. The experimental analysis shows all of the study pumps can operates in turbine mode at higher flow rate and head without any mechanical modification.

The advancement of numerical analysis in Computational Fluid Dynamics (CFD) offers a reliable by means of virtually analysing complex fluid flows inside a pump. For PAT studies, the performance curve parameters consist of the operating head, flow rate, hydraulic efficiency and brake horse power can be investigate numerically. This will reduce investigation time and cost by a significant margin. A well-defined CFD methodology on centrifugal pumps running in turbine mode has been reported in a number of studies. However, the diverse geometric profiles and operating conditions require discrete analyses of a specific pump.

Yang et al. (2012) had conducted CFD analysis on radial flow pump running at a range of rotational speed. The study shows numerical predicted analysis is at good agreement with experimental result [8]. Rawal and Kshirsagar (2007) conducted numerical simulation on a mixed flow pump running in turbine mode emphasising good quality mesh and suitable turbulence model to improve accuracy of the results [9]. Derakhshan and Nourbakhsh (2008) suggested geometric simplification between impeller and volute flow domain poor simulation accuracy, highlighting the importance of interface between flow domain between pump stator and rotor [10].

In this study, simulations are performed on a centrifugal pump running in turbine mode using a commercial CFD software. The simulation results are presented in performance curve reflecting the PAT characteristic running at a defined range of flow coefficient. Moreover, the hydraulic characteristics when the pump interacts with the rotational impeller are also presented.

\section{Research Methodology}

ANSYS CFX 14.0 software was used for CFD modelling and numerical computations. For pump mode operation, the predicted head coefficient, flow coefficient and power coefficient were compared with the performance curve provided by the pump manufacturer in order to verify the accuracy of the model. Following this, simulations were carried out for inverse flow operation. The rotational speed was set at 1450 and 1550 RPM for pump and turbine mode, respectively. It is worth to mention, the rotational speed was selected in order to match the rotational speed of the four-pole induction generator typically direct coupled with the PAT.

Pump Specifications. Euroflo centrifugal pump with back pull feature was selected for the CFD model. The dimensions of the pump are in accordance with the EN733:1995/DIN24255 standard. The impeller has a diameter of $214.0 \mathrm{~mm}$ and the flange diameter at the inlet and outlet is $50.0 \mathrm{~mm}$ and $65.0 \mathrm{~mm}$, respectively. The pump power rating is $2.2 \mathrm{~kW}$ and rotational speed of $1450 \mathrm{RPM}$. The best efficient point (BEP) is $65.0 \%$ at head and flow rate of $14.0 \mathrm{~m}$ and $8.1 \mathrm{1} / \mathrm{s}$, respectively. The non-dimensional specific speed, $\mathrm{N}_{\mathrm{s}}$ is 70 units.

CAD Model. The geometry of the pump consisting of the impeller, volute and draft tube is shown in Fig. 1(a). The flow domain was simplified by excluding the front and back casings in the computational flow domain. All flow gaps at the wear ring as well as between the impeller and volute were excluded from the simulation to reduce the effects of boundary condition on the inner flow.

Mesh Generation. Unstructured tetrahedral mesh was selected for all flow regions as it was proved suitable to resolve the complex geometric topology in order to capture the flow physics. The mesh element face and size were set at $1.5 \mathrm{~mm}$. Hence, the number of elements for the volute and impeller was obtained to be 450381 and 476601, respectively, whereas the number of elements for the draft tube was 167226 elements. Mesh independence analysis was performed to evaluate the rate of convergence, solution accuracy and computational time. The convergence criterion was set at a total of 227933 nodes, which was satisfactory to attain mesh independence. The mesh topography for all flow domains is shown in Fig. 1(b). 


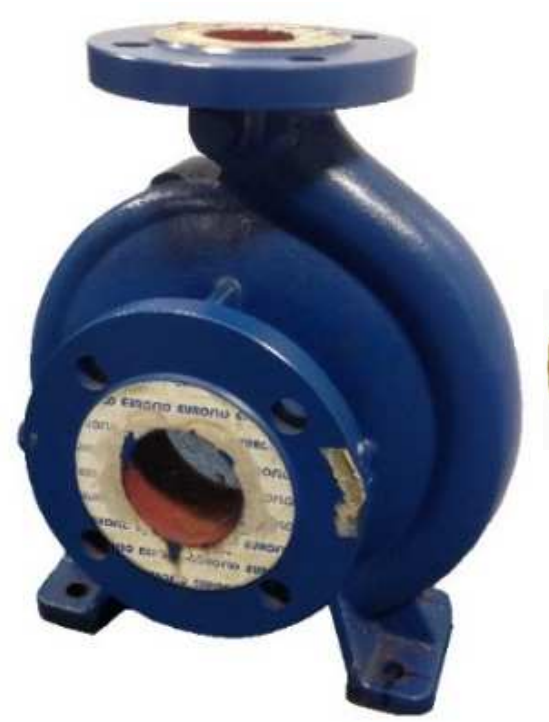

(a)

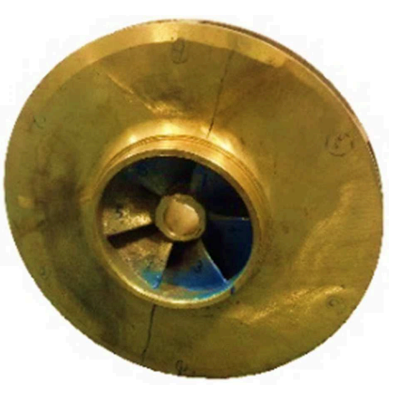

(b)

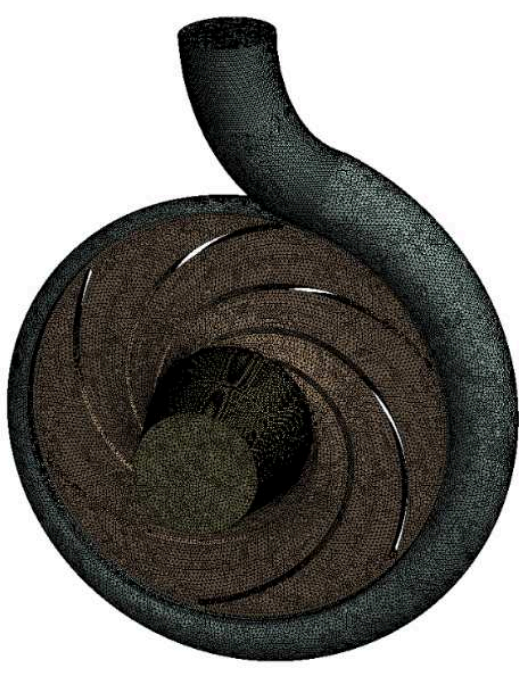

Fig. 1 (a) Pump housing and impeller (b) CFD flow domain and mesh topography

Boundary Condition and Solver. Water with density of $1000 \mathrm{~kg} / \mathrm{m}^{3}$ at $25^{\circ} \mathrm{C}$ was chosen as the working fluid in this study. The boundary conditions were set as follows. The mass flow rate at the inlet was set constant while the static pressure at the outlet was set at $1 \mathrm{~atm}$. The mass flow rate was varied to obtain the pump and turbine performance curves. The CFD analysis was set to compute in steady-state conditions. The flow rate was varied between 0.7 and $1.3 \mathrm{Q}_{\mathrm{BEP}}$ for both pump and turbine modes to prevent convergence issues due to software limitations [11]. The inner wall of the PAT was set as no-slip wall and the wall roughness was set at 100 microns. Standard k- $\epsilon$ turbulence was used as the turbulence model since it is widely used in centrifugal pump simulations.

The governing equations relevant to three-dimensional, incompressible flows in ANSYS CFX software are established based on unsteady Navier-Stokes equations. The governing equations are the fundamental principles of fluid dynamics and comprise the continuity, momentum and energy equation.

\section{Results and Discussion}

The characteristic curves of the pump are represented by non-dimensionless parameters, namely, flow coefficient, head coefficient, power coefficient and efficiency coefficient.

Pump Mode. For pump mode, the performance curve obtained from simulations was compared with the performance curve given by the pump manufacturer. The pump and turbine performance curve are shown in Fig. 2. It can be observed that there is good agreement between the simulation results and the manufacturer performance curve. The BEP of the pump is achieved at head and flow rate of $12.92 \mathrm{~m}$ and $8.0 \mathrm{l} / \mathrm{s}$, while the efficiency is recorded at $72.63 \%$. The difference between the predicted values and those given in the manufacturer performance curve may be attributed to mechanical losses due to disc friction and leakage at the front and back casings of the impeller which are excluded from the computation domain. This explained why the power coefficient obtained in the simulation is less than that given in the manufacturer data sheet. 

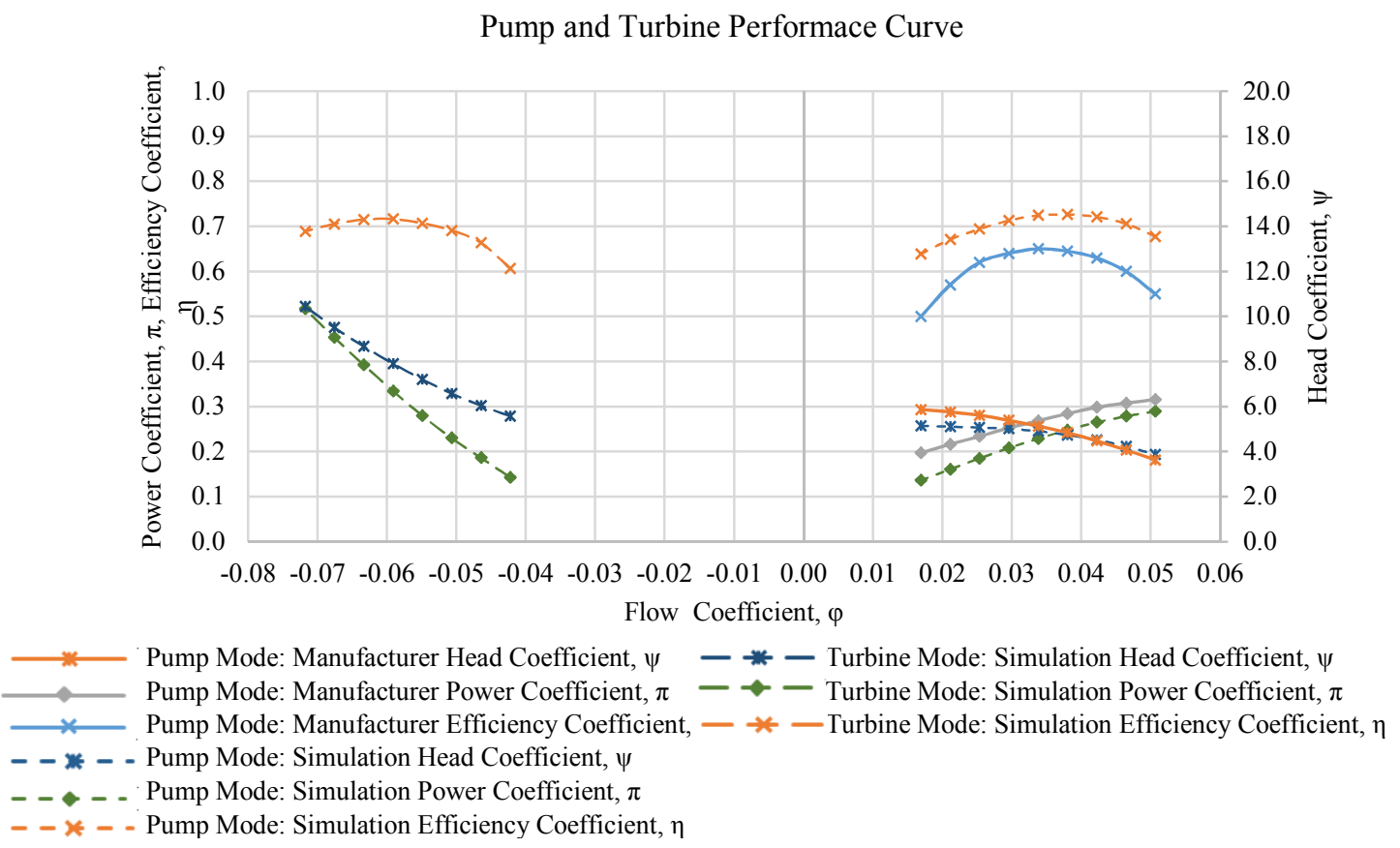

Fig. 2: Dimensionless performance curve in pump and turbine modes running at 1450 and 1550 RPM
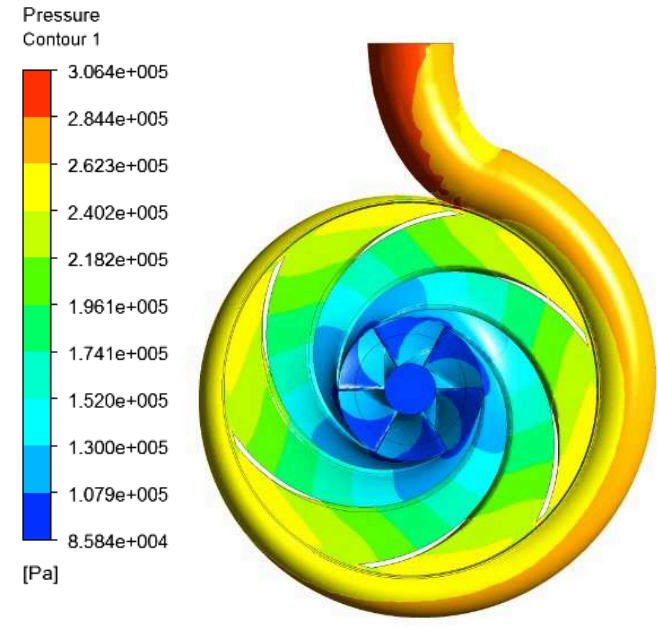

(a)

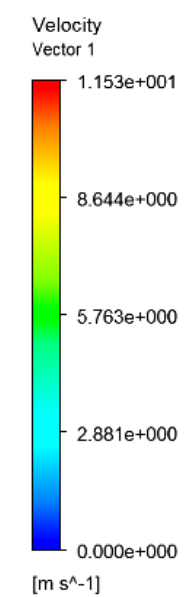

(b)

Fig. 3: Simulation results for a flow coefficient of -0.0591

(a) Pressure distribution for all flow domains (b) Velocity vector of flow inside the impeller

Turbine Mode. It can also be observed that the BEP is attained at a higher head and flow rate (21.55 $\mathrm{m}$ and $14.0 \mathrm{1} / \mathrm{s}$, respectively) when the pump operates in turbine mode. The highest efficiency for turbine mode is obtained at $71.62 \%$, which is certainly lower than the pump mode.

The local flow characteristics inside the pump are shown in Fig. 3. The pressure force and flow velocity are the driving mechanisms inside the pump volute and impeller. The pressure distribution inside the impeller and volute is shown in Fig. 3(a) and it can be seen that the pressure force decreases gradually along volute path. As water enters into the impeller, the pressure decreases along the impeller channel due to conversion of energy from fluid energy to rotational mechanical energy. The pressure distribution between the pressure surface and suction surface (beginning from the outer diameter of the impeller) becomes uniform at the exit of the impeller.

The velocity vector inside the impeller flow domain is shown in Fig. 3(b). The velocity vector of the water entering the pump inlet reveals no sudden change in flow patterns, with a smooth velocity transition. The water is directed by the curvature of the volute wall (which has a spiral shape) and thus maintains an even flow path to the impeller. 


\section{Conclusion}

CFD simulations of an end suction centrifugal pump operating at a specific speed of 70 units have been described in this paper. Inverse flow tests were performed at a rotational speed of 1450 and 1550 RPM in order to match the specifications of a four-pole induction generator. The simulation results reveal that the centrifugal pump attains BEP at a higher head and flow rate and exhibits lower efficiency when running in turbine mode. It can be concluded that the prediction of the PAT performance using CFD is viable. However, it is recommended that further work is needed to validate the accuracy of the results by means of experiments. In addition, simulations should be carried out at various specific speeds of the pump in order to clarify the accuracy of the model for a vast range of operating conditions.

\section{Acknowledgement}

This research was supported by the Centre of Excellence for Renewable Energy (CoERE), University Malaysia Sarawak (UNIMAS) under Grant no. UNIMAS/CoERE/2014/Grant (01).

\section{References}

[1] S. Chuenchooklin, "Development of Pico hydropower Plant for Farming Village in Upstream Watershed, Thailand," presented at the Agricultural Development within the Rural-Urban Continuum, 2006.

[2] M. Arriaga, "Pump as Turbine - A Pico-Hydro Alternative in Lao People's Democratic Republic," Renewable Energy, vol. 35, pp. 1109-1115, 2010.

[3] M. A. Ismail, A. K. Othman, S. Islam, and H. Zen, "End Suction Centrifugal Pump Operating in Turbine Mode for Microhydro Applications," Advances in Mechanical Engineering, vol. 2014, p. 7, 2014.

[4] S. Derakhshan and A. Nourbakhsh, "Experimental Study of Characteristic Curves of Centrifugal Pumps Working as Turbines in Different Specific Speeds," Experimental Thermal and Fluid Science, vol. 32, pp. 800-807, 2008.

[5] N. Raman, I. Hussein, K. Palanisamy, and B. Foo, "An Experimental Investigation of Pump as Turbine for Micro Hydro Application," presented at the 4th International Earth on Energy and Environment, 2013.

[6] J. Fernández, E. Blanco, J. Parrondo, M. Stickland, and T. Scanlon, "Performance Of a Centrifugal Pump Running in Inverse Mode," Proceedings of the Institution of Mechanical Engineers, Part A: Journal of Power and Energy, vol. 218, pp. 265-271, 2004.

[7] H. Nautiyal, Varun, A. Kumar, and S. Yadav, "Experimental Investigation of Centrifugal Pump Working as Turbine for Small Hydropower System," Energy Science and Technology, vol. 1, 2011.

[8] S. S. Yang, S. Derakhshan, and F. Y. Kong, "Theoretical, numerical and experimental prediction of pump as turbine performance," Renewable Energy, vol. 48, pp. 507-513, 2012.

[9] S. Rawal and J. Kshirsagar, "Numerical Simulation on a Pump Operating in a Turbine Mode," in Proceedings of the 23rd International Pump Users Symposium, 2007, pp. 21-27.

[10] S. Derakhshan and A. Nourbakhsh, "Theoretical, Numerical and Experimental Investigation of Centrifugal Pumps in Reverse Operation," Experimental Thermal and Fluid Science, vol. 32, pp. 1620-1627, 2008.

[11] M. Sedláŕ, J. Šoukal, and M. Komárek, "CFD Analysis, Multistage Radial-Flow Pump, Turbine Regime," Association for Engineering Mechanics, vol. 16, pp. 1802-1484, 2009. 